\title{
ÚNG DỤNG THỬ NGHIỆM ẢNH VỆ TINH SPOT6 TRONG CÔNG TÁC THÀNH LẬP MÔ HìNH SỐ ĐỊA HÌNH (DSM)
}

\author{
PHAM MINH HẢl, TRỊNH THI! NGỌC BÍCH \\ Viện Khoa học Đo đạc và Bản đồ
}

\section{Tóm tắt}

SPOT6 và SPOT7 là hai vệ tinh độ phân giải cao trong các thế hệ vệ tinh SPOT. Độ phân giải không gian của các vệ tinh này đã được nâng lên 1,5m so với 2,5m của SPOT 5, đây là vệ tinh quang học SPOT với nhiều cải tiến về kỹ thuật và khả năng thu nhận ảnh. Việc ứng dụng nguồn tư liệu độ phân giải cao này trong các hoạt động nghiên cứu và xây dựng mô hình số độ cao phục vụ mục đích thành lập và cập nhật phần địa hình chưa được tiến hành phổ biến. Mục tiêu của bài báo giới thiệu công tác thử nghiệm thành lập thành lập mô hình số địa hình (DSM) sử dụng cặp ảnh lập thể SPOT6 phục vụ công tác định hướng sử dụng nguồn dữ liệu này trong các ứng dụng thành lập và cập nhật dữ liệu phần địa hình. Nhóm nghiên cứu sử dụng phần mềm PCI Geomatica 2015 và phần mềm Google Earth để tiến hành thành lập và đánh giá độ chính xác của mô hình số bề mặt của khu vực thực nghiệm.

\section{Giới thiệu}

Công nghệ thành lập mô hình số địa hình (DSM) bằng công nghệ viễn thám bắt đầu được sử dụng nhiều vào cuối những năm 1950. DSM được ứng dụng khá rộng rãi trong nhiêu lĩnh vực như đo đạc bản đồ, hỗ trợ lập các mô hình về các nguy cơ xói mòn đất, lũ lụt, nghiên cứu hướng gió và các mô hình phát tán ô nhiễm, các hoạt động quân sự như dẫn đường cho tên lửa, mô phỏng chiến sự.v.v... Các công nghệ thành lập DSM dần được cải tiến cùng với sự phát triển của các công nghệ bay chụp ảnh viễn thám. Từ năm 2012 đến 2014, các vệ tinh SPOT6 và SPOT7 lần lượt được phóng lên bầu trời. Độ phân giải không gian của kênh panchromatic và kênh phổ của các vệ tinh này đã được nâng lên $1,5 \mathrm{~m}$ và $6 \mathrm{~m}$ so với $2,5 \mathrm{~m}$ và $10 \mathrm{~m}$ của SPOT 5 , đây là thế hệ mới của loạt vệ tinh quang học SPOT với nhiều cải tiến về kỹ thuật và khả năng thu nhận ảnh. Đây là nguồn dữ liệu ảnh vệ tinh độ phân giải cao phục vụ các mục đích về thành lập và cập nhật thông tin về địa hình của các loại bản đồ nền và bản đồ chuyên đề. Nghiên cứu về khảo sát các đặc tính kỹ thuật và đánh giá khả năng ứng dụng của ảnh SPOT6 trong giám sát tài nguyên và môi trường đã được thực hiện trong nghiên cứu của Hồ Thị Vân Trang (2015). Tuy nhiên, cho tới nay chưa ghi nhận một nghiên cứu nào ở nước ta nghiên cứu về ứng dụng ảnh lập thể của vệ tinh SPOT6 trong thành lập mô hình số độ cao.

Do đó, mục tiêu của bài báo giới thiệu công tác khảo sát, thử nghiệm thành lập thành lập mô hình số địa hình (DSM) sử dụng cặp ảnh lập thể SPOT6 phục vụ công tác định hướng sử dụng nguồn dữ liệu này trong các ứng dụng thành lập và cập nhật dữ liệu phần địa hình. Với sự hạn chế về nguồn dữ liệu chụp ở Việt Nam, nhóm nghiên cứu tiến hành sử dụng cặp ảnh lập thể SPOT6 được chụp tại Thành phố Melbourne, Úc làm dữ liệu đầu vào (xem hình 1). Phần mềm $\mathrm{PCl}$ Geomatica 2013 và phần mềm Google Earth được sử dụng để tiến hành thành lập và đánh giá độ chính

Ngày nhận bài: 07/6/2017, ngày chuyển phản biện: 09/6/2017, ngày chấp nhận phản biện: 14/6/2017, ngày chấp nhận đăng: 16/6/2017 
xác của mô hình số bề mặt của khu vực thực nghiệm.

\section{Dữ liệu thực nghiệm}

Nghiên cứu được thực nghiệm với cặp ảnh lập thể SPOT6. Vệ tinh SPOT6 có khả năng chụp lập thể trên cùng 1 dải ảnh nhờ chế độ chụp ảnh bằng hai đầu chụp bố trí trước, sau, và có thể điều chỉnh góc chụp. Bên cạnh đó, với sự thay đổi của góc nghiêng của đầu chụp mà chế độ chụp lặp của vệ tinh có thể thay đổi từ 26 ngày (ứng với góc nghiêng 5 độ) đến 2 ngày (ứng với góc nghiêng 45 độ). (xem bảng 1, hình 2)

3. Quy trình công nghệ thành lập DSM và thực nghiệm trên phần mềm $\mathrm{PCl}$ Geomatica 2013

Nhóm nghiên cứu tiến hành công tác thực nghiệm thành lập DSM khu vực thử nghiệm bằng cặp ảnh lập thể SPOT6 trên phần mềm $\mathrm{PCl}$ Geomatica 2015 với quy trình thực hiện được mô tả trên hình 2. Cơ sở khoa học của phương pháp dựa trên nguyên lý đo vẽ ảnh hàng không (Trần Tuấn Ngọc và nnk, 2012). (xem hình 3)

Bước 1: Tiến hành nhập ảnh

Cặp ảnh lập thể SPOT6 được nhập vào hệ thống Geomatica 2013. Việc tạo mới một project trong phiên làm việc đầu tiên là phải chọn hệ thống phép chiếu cho khu vực đo vẽ. Phần mềm tự động tính toán từ các tham số quỹ đạo dựa vào các thông số metadata của ảnh để lựa chọn cặp ảnh chính - phụ. Ảnh chính sẽ là ảnh gần khu vực quét ảnh hơn so với ảnh phụ. Các điểm khống chế ảnh (GCP) cũng được đo trong quá trình định hướng ảnh nhằm xác định mối quan hệ giữa hệ tọa độ không gian $3 D$ và hệ tọa độ ảnh $2 \mathrm{D}$ cũng như để tính toán hiệu chỉnh sai số quỹ đạo của vệ tinh. Do điều kiện hạn chế trong công tác thực địa nên các điểm khống chế ngoại nghiệp được nhóm đề tài chọn dựa trên tham khảo từ nguồn phần mềm Google Earth được mô tả trên hình 5. (xem bảng 2, hình 4, hình 5)

Các điểm khống chế được chọn trong phần thực nghiệm phải thỏa mãn các yêu cầu:

- Các địa vật được chọn được nhìn rõ nét trên ảnh (góc nhà, cầu);

- Vị trí các điểm khống chế được chọn ưu tiên tại chỗ giao nhau các địa vật hình tuyến;

- Số lượng điểm khống chê được chọn là 11 điểm được bố trí đều trên ảnh.

Với độ phân giải không gian 1.5m sau khi trộn ảnh, cùng với độ phân giải bức xạ của ảnh SPOT6 là 12 bit, các điểm khống chế được quan sát rõ ràng trên ảnh, do đó độ chính xác về vị trí của điểm khống chế trên ảnh được chọn được nâng cao. Đây là ưu điểm nổi bật của ảnh SPOT6 khi sử dụng để chọn điểm khống chế trên ảnh so với các loại ảnh khác như SPOT5, VNRed-Sat1 trong cùng ứng dụng. (xem hình 6 )

Bước 2: Định hướng tương đối mô hình lập thể

Đây là bước xử lý nhằm định hướng tương đối ảnh phụ so với ảnh chính của mô hình lập thể. Các điểm nối (tie-points) đo được trên mô hình sẽ sử dụng để tính chuyển bằng phương pháp chuyển đổi

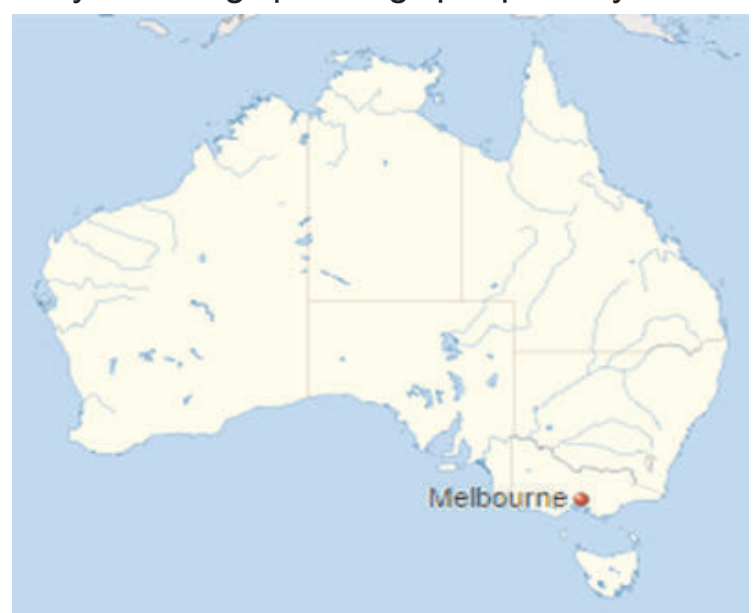

Hình1: Khu vực nghiên cứu (Nguồn: https://en.wikipedia.org/wiki/Australia) 


\section{Nghiên cúu - Üng dụng}

Bảng 1: Các thông số kỹ thuật của ảnh SPOT6 (Nguồn: Hồ Thị Vân Trang, 2015)

\begin{tabular}{|l|l|}
\hline \multicolumn{1}{|c|}{ Đặc điếm } & \multicolumn{1}{c|}{ Thông số kỹ thuật } \\
\hline \multirow{3}{*}{ Kênh phổ } & Blue $(0.455 \mu \mathrm{m}-0.525 \mu \mathrm{m})$ \\
& $\begin{array}{l}\text { Green }(0.530 \mu \mathrm{m}-0.590 \mu \mathrm{m}) \\
\text { Red }(0.625 \mu \mathrm{m}-0.695 \mu \mathrm{m}) \\
\\
\text { Near-Infrared }(0.760 \mu \mathrm{m}-0.890 \mu \mathrm{m})\end{array}$ \\
\hline Độ phân giải & $\begin{array}{l}\text { Panchromatic }-1.5 \mathrm{~m} \\
\text { Multispectral - } 6.0 \mathrm{~m}(\mathrm{~B}, \mathrm{G}, \mathrm{R}, \mathrm{NIR})\end{array}$ \\
\hline Kích thước ảnh & $60 \mathrm{Km}$ ở vị trí trực tâm (Nadir) \\
\hline Độ lặp & 26 ngày \\
\hline Thời gian chụp & \\
\hline
\end{tabular}

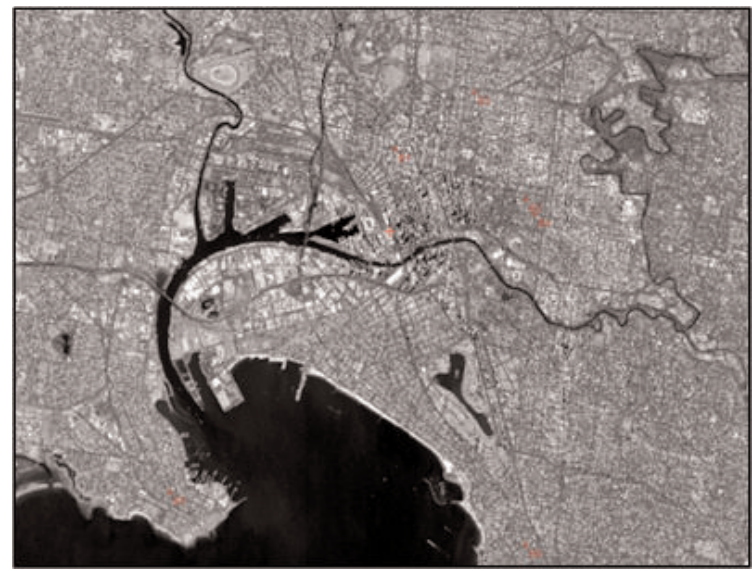

Hình trái

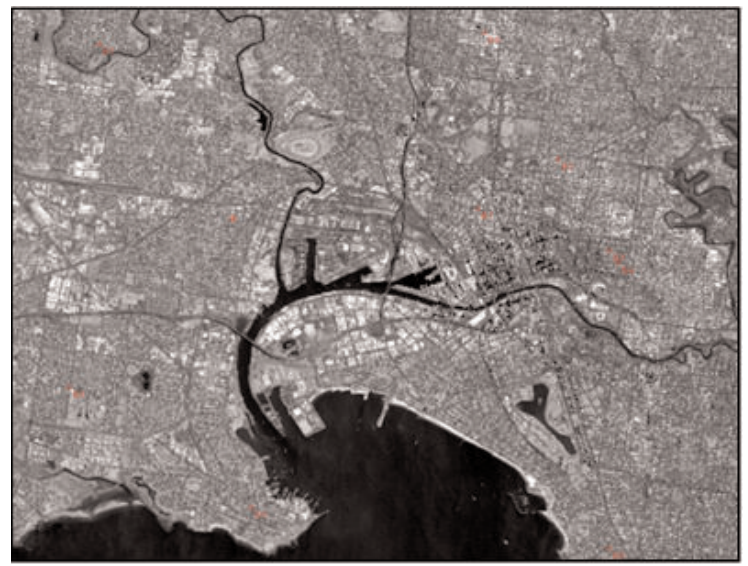

Hình phải

Hình 2: Cặp ảnh lập thể SPOT6 được sử dụngtrong nghiên cứu

Affine. Khi tính toán bình sai, các điểm có sai số vượt quá hạn sai cho phép được kiểm tra lại trên mô hình. Kết quả bình sai phần lớn đáp ứng yêu cầu về độ chính xác với độ chính xác sau bình sai nhỏ hơn 1 pixel (2 mét) ở khu vực thành thị và nhỏ hơn 2 pixel (4 mét) ở khu vực có núi. Các tham số được tính toán trong bước này như thị sai tối đa theo trục $x$ và tối thiểu theo trục $y$ được sử dụng để hiệu chỉnh các tham số khớp ảnh cho phù hợp.

\section{Bước 3: Quá trình khớp ảnh}

Quá trình khớp ảnh được thực hiện bằng phương pháp tương quan để tìm kiếm các điểm ảnh cùng tên trên cặp ảnh chính và phụ. Sử dụng cấu trúc hình học epipolar để tính toán sẽ cho phép thu nhỏ kích thước cửa sổ tìm kiếm nhằm tránh các kết quả sai trong quá trình khớp ảnh cũng như giảm đáng kể thời gian tính toán. Kết quả nhận được sau bước xử lý này là các giá trị thị sai của tất cả các phần tử ảnh được tính theo trục tọa độ $X$ và $Y$. Các thị sai này được lưu giữ dưới dạng thức các file ảnh gọi là ảnh thi sai $X$ (X parallax image) và ảnh thị sai $Y$ ( $Y$ parallax image). Đồng thời, các hệ số tương quan của từng phần tử ảnh được tính trong quá trình xử lý cũng được lưu giưr trong file ảnh gọi là ảnh tương quan (correlation image).

\section{Bước 4: Tính giá trị độ cao}

Tính toán giá trị tọa độ không gian 3D sử dụng giá trị thị sai độ cao và các tham số mô hình lập thể đã được tính trong các bước xử lý trước đó bằng phương pháp tính giao hội không gian theo nguyên tắc số trung phương nhỏ nhất. Kết quả nhận được sau bước xử lý này là mô hình số bề mặt (DSM) dưới dạng raster với giá trị giãn cách lưới đều (grid spacing) hay nói cách khác kích 
Bảng 2: Tọa độ các điểm khống chế được sưr dụng

\begin{tabular}{|c|c|c|c|c|}
\hline \multirow{2}{*}{ Điểm } & \multicolumn{3}{|c|}{ Toạ độ } \\
\cline { 3 - 5 } & & $\mathbf{X}$ & $\mathbf{Y}$ & $\mathbf{Z}$ \\
\hline 1 & B1 & 319883.846 & 5814009.838 & 43.504 \\
\hline 2 & B2 & 322712.645 & 5813146.521 & 34.726 \\
\hline 3 & B3 & 321578.401 & 5815160.540 & 31.593 \\
\hline 4 & B4 & 322914.637 & 5812867.987 & 27.150 \\
\hline 5 & B5 & 323687.315 & 5815748.160 & 12.132 \\
\hline 6 & B6 & 311450.470 & 5815786.602 & 15.286 \\
\hline 7 & B7 & 315197.665 & 5807243.519 & 42.663 \\
\hline 8 & B8 & 328390.280 & 5818107.106 & 42.173 \\
\hline 9 & B9 & 322690.143 & 5806603.142 & 18.542 \\
\hline 10 & B10 & 312085.546 & 5809730.753 & 32.630 \\
\hline 11 & B11 & 319955.505 & 5817954.925 & 62.301 \\
\hline
\end{tabular}

thước pixel được xác định phù hợp với các tiêu chuẩn quy định kỹ thuật ở tỷ lệ bản đồ cần thành lập.

Kết quả nội suy các giá trị độ cao của mô hình số bề mặt được thể hiện trên hình 7 . (xem hình 7)

\section{4. Đánh giá độ chính xác của kết quả nghiên cứu}

Kết quả thực hiện của nghiên cứu được đánh giá độ chính xác bằng phương pháp so sánh độ cao giữa các điểm cùng vị trí trên sản phẩm và trên ảnh của phần mềm Google Earth. (xem hình 8)

Kết quả so sánh độ chính xác về độ cao DSM được chiết xuất từ cặp ảnh lập thể SPOT6 dưới đây: (xem bảng 3)

Qua kết quả đánh giá độ chính xác, nhóm nghiên cứu nhận thấy: Với độ phân giải không gian $1.5 \mathrm{~m}$ sau khi trộn ảnh, cùng với độ phân giải bức xạ của ảnh SPOT6 là 12 bit, công tác lựa chọn điểm khống chế ảnh phục vụ công tác định hướng ảnh được thực hiện dễ dàng và chính xác ở trên ảnh, tuy công tác thực địa đo vẽ khống chế ảnh chưa được thực hiện do hạn chế về khoảng cách địa lý. Hình 9 cho thấy các điểm độ cao của mô hình số bề mặt của khu vực thực nghiệm có dáng tương đồng với xu hướng của các điểm độ cao được tham chiếu từ phần mềm Google Earth. (xem hìn 9)

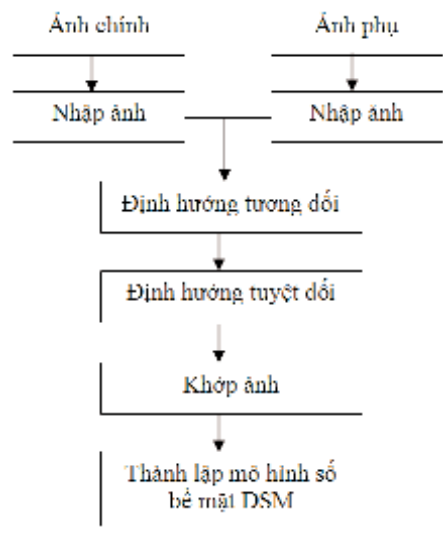

Hình 3: So đồ quy trình thành lập DSM từ ảnh Spot6

\section{Kết luận}

Nhìn chung, kết quả mô hình số bề mặt khu vực thành phố Melbourne, Úc được thành lập bán tự động từ quy trình ở phần 2 đã mô tả được dáng địa hình khu vực thực nghiệm với sai số nằm trong hạn sai cho phép nhỏ hơn 1 pixel (2 mét) ở khu vực thành thị và nhỏ hơn 2 pixel (4 mét) ở khu vực có núi. Đây là kết quả có thể tham khảo cho các nghiên cứu ứng dụng sử dụng ảnh SPOT6 phục vụ mục đích quan trắc lớp phủ bề mặt và thành lập mô hình số bề mặt các khu vực ngoài biên giới khi công tác đo vẽ điểm khống chế bị hạn chế. Việc lựa chọn điểm khống chế được chưa có sự đối soát ngoài thực địa do vậy một số khu vực độ cao lớn hơn mức thực tế là do quá trình nội suy theo thuật toán Nearest Neignbour tại các vị trí địa vật xa vị trí chọn điểm khống chế làm cho độ cao khu vực này bị sai lệch đi. Kết quả nghiên cứu có tính khoa học và ứng dụng cao, quy trình thực hiện với thao tác sử dụng đơn giản đảm bảo công tác thành lập mô hình số bề mặt bằng cặp ảnh lập thể SPOT6 đạt độ chính xác cao, tiết kiệm thời gian và hiệu quả về kinh tế. $\bigcirc$

\section{Tài liệu tham khảo:}

[1]. Hồ Thị Vân Trang và nnk (2015). Nghiên cứu đánh giá khả năng ứng dụng ảnh vệ tinh SPOT6 trong giám sát tài nguyên môi trường ở Việt Nam. Đề tài 

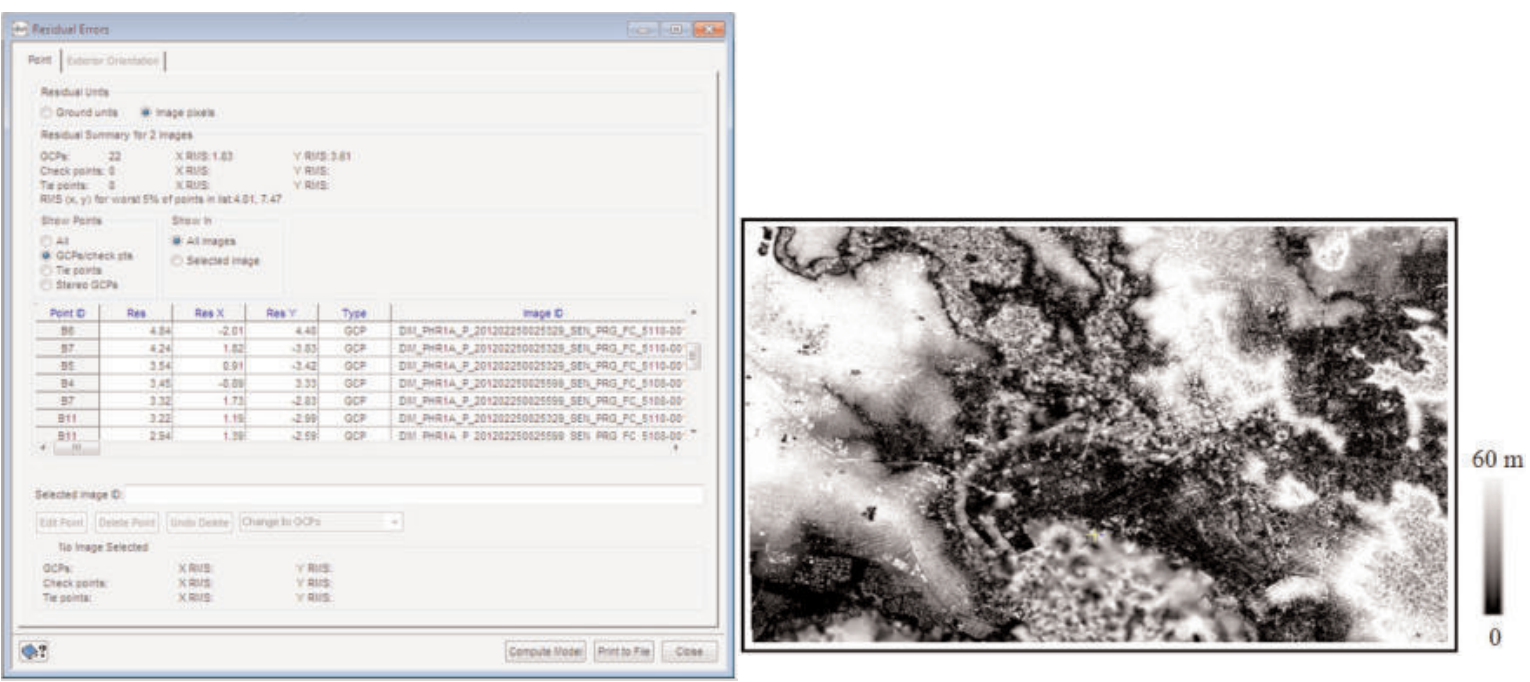

Hình 4: Bảng kết quả bình sai bằng phần mềm PCI

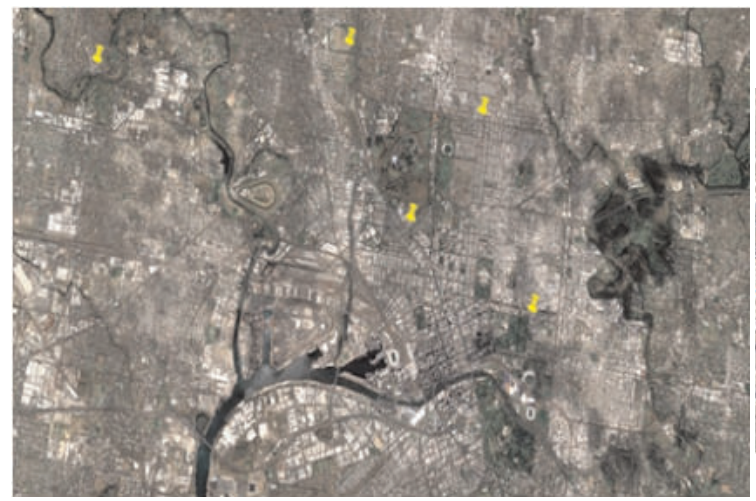

Hình 7: Kết quả nội suy mô hình số địa hình khu vực thử nghiệm

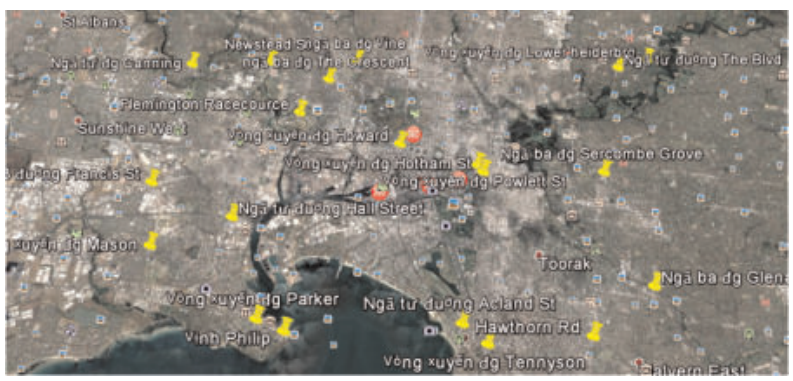

Hình 5: Tham khảo lựa chọn điểm khống chế trên phần mềm Google Earth tương ứng với các điểm GCP lựa chọn trên ảnh lập thể SPOT6

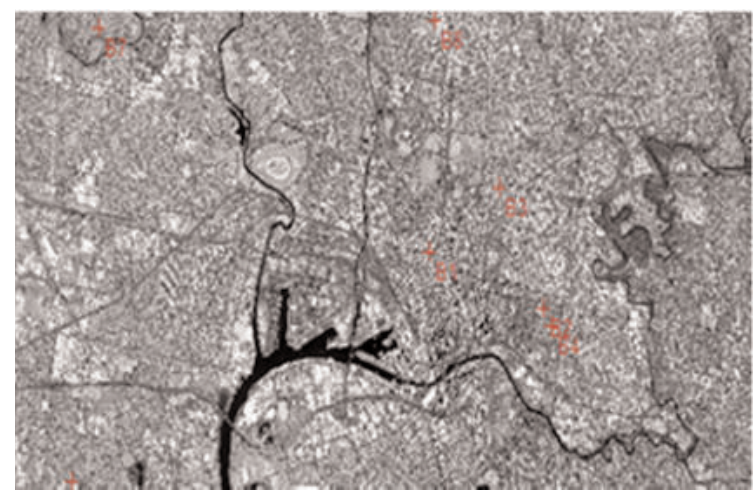

Hình 6: Công tác chọn điểm khống chế trên ảnh SPOT6

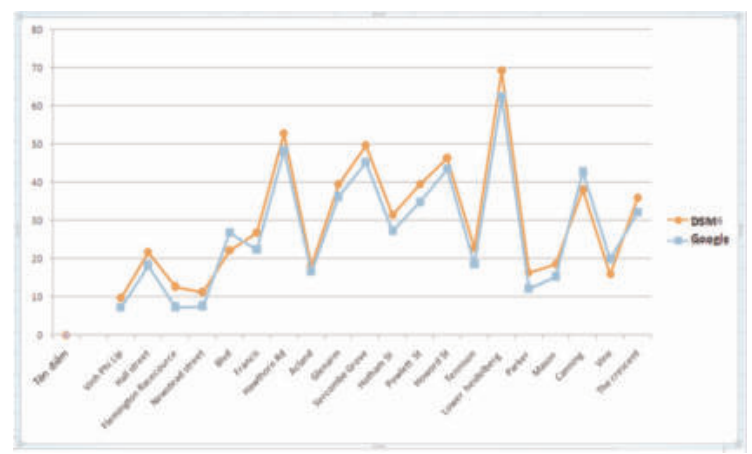

Hình 9: So sánh một số điểm độ cao từ sản phẩm và độ cao từ Google 
Bảng 3: So sánh các điểm độ cao trên sản phẩm và trên Google Earth

\begin{tabular}{|r|l|c|c|c|c|c|}
\hline TT & \multicolumn{1}{|c|}{ Điểm } & $\mathrm{X}$ & $\mathrm{Y}$ & $\begin{array}{c}\text { Độ cao trên } \\
\text { Google Earth }\end{array}$ & $\begin{array}{c}\text { Độ cao trên } \\
\text { sản phẩm }\end{array}$ & Độ lệch \\
\hline 1 & Vịnh Phi Líp & 316142.616 & 5806845.803 & 7.245 & 9.725 & 2.480 \\
\hline 2 & Hallstreet & 313981.042 & 5810969.603 & 18.208 & 21.695 & 3.487 \\
\hline 3 & $\begin{array}{l}\text { Flemington } \\
\text { Racecource }\end{array}$ & 316076.035 & 5815362.029 & 7.324 & 12.712 & 5.388 \\
\hline 4 & Newstead street & 314734.281 & 5817688.190 & 7.552 & 11.245 & 3.693 \\
\hline 5 & Blvd & 328298.103 & 5817557.646 & 26.705 & 22.185 & -4.520 \\
\hline 6 & Francis & 310936.668 & 5812316.843 & 22.312 & 26.871 & 4.559 \\
\hline 7 & Hawthorn Rd & 326312.990 & 5806782.724 & 48.243 & 52.813 & 4.570 \\
\hline 8 & Acland & 322031.781 & 5807185.374 & 16.522 & 18.223 & 1.711 \\
\hline 9 & Glenarm & 328552.294 & 5808567.773 & 36.224 & 39.456 & 3.232 \\
\hline 10 & Sercombe Grove & 327288.824 & 5812910.887 & 45.211 & 49.788 & 4.577 \\
\hline 11 & Hotham St & 322914.637 & 5812867.987 & 27.150 & 31.568 & 4.418 \\
\hline 12 & Powlett St & 322712.645 & 5813146.521 & 34.726 & 39.456 & 4.730 \\
\hline 13 & Howord St & 319883.846 & 5814009.838 & 43.504 & 46.378 & 2.874 \\
\hline 14 & Tennison & 322690.143 & 5806603.142 & 18.542 & 22.886 & 4.344 \\
\hline 15 & Lower heidelberg & 319955.505 & 5817954.925 & 62.301 & 69.247 & 6.946 \\
\hline 16 & Parker B5 & 323687.315 & 5815748.160 & 12.132 & 16.348 & 4.216 \\
\hline 17 & Mason B6 & 311450.470 & 5815786.602 & 15.286 & 18.682 & 3.396 \\
\hline 18 & Canning B7 & 315197.665 & 5807243.519 & 42.663 & 38.056 & -4.607 \\
\hline 19 & Vine & 318150.976 & 5817772.632 & 19.825 & 15.987 & -3.838 \\
\hline 20 & Thecescent & 317093.375 & 5816860.723 & 32.114 & 35.987 & 3.873 \\
\hline
\end{tabular}

nghiên cứu khoa học cấp cơ sở của Bộ Tài nguyên và Môi Ttrường;

[2]. Hennig, S.D., Koppe, W., Kiefl, N., Janoth, J., Duering, R. 2008. Digital Elevation Modeling using TerraSAR-X Radargrammetry.

[3]. Michele Crosetto, Fernando Pérez Aragues (2000). Radargrammetry and SAR interferometry for DEM generation: validation and data fusion.

[4]. Trần Tuấn Ngọc và nnk, 2012, Nghiên cứu ứng dụng ảnh vệ tinh RADAR độ phân giải cao trong thành lập mô hình số độ cao và kiểm kê đảo. Đề tài nghiên cứu khoa học cấp Bộ, Bộ Tài nguyên và Môi trường. $\mathrm{O}$

\section{Summary}

\section{Using SPOT6 stereo satellite imagery in making digital surface models (DSM)}

\section{Pham Minh Hai, Trinh Thi Ngoc Bich, Vietnam Institute of Geodesy and Cartography}

SPOT6 and SPOT7 are new generations of SPOT high spatial resolution images. The spatial resolution of these satellites has been improved to $1.5 \mathrm{~m}$ compared to $2.5 \mathrm{~m}$ of SPOT5, moreover many improvements in technology and image acquisition capabilities were developed in these satellies. These high-resolution images play very important role for the purpose of establishing and updating terrain maps, however they have not yet been widely applied in Vietnam yet. The objective of this paper is to investigate the potential of SPOT6 stereo satellite imagery in making digital terrain model (DSM). PCI Geomatica 2015 software was used to extract DSM and Google Earth software was used to evaluate the accuracy of the experimental result. $\mathrm{O}$ 